\title{
Dyspnoea, cough, edema: Heart failure or what else?
}

\author{
Luca Morolli, Davide Lazzarini", Giuseppa Micali, Giorgio loli
}

Third Department of Internal Medicine, Azienda Unita Sanitaria Locale di Rimini, Rimini, Italy;

*Corresponding Author: davide.lazzarini@auslrn.net

Received 23 November 2011; revised 7 January 2012; accepted 14 January 2012

\begin{abstract}
We present the case of a 55-year-old man admitted for dyspnea, cough and edema of the legs and feet. The patient, a smoker with psoriasis at hands and feet noted the comparison, three years ago, of marked thickening and yellow discoloration of the nails. The chest X-ray showed a right-sided pleural effusion with parenchymal atelectasis. The cytologic examination of pleural fluid revealed mesothelial cells with inflammatory changes. The bacterial culture of the pleural fluid was negative and pleural biopsies revealed fibrosis. To reduce dyspnoea and formation of pleural fluid, was performed a pleurodesis by talcage. The patient was discharged with itraconazole, high dose vitamin $\mathrm{E}$ and zinc supplements as home therapy. The correct diagnosis was not easily achieved because the patient had symptoms and signs referable to other diseases as heart failure or venous insufficiency or psoriasis.
\end{abstract}

Keywords: Samman-Emerson Syndrome; Yellow Nail Syndrome; Limphedema; Pleural Effusion

\section{INTRODUCTION}

Samman and White [1] first described the Yellow Nail Syndrome, characterized by Emerson [2] in the classic triad: yellow discoloured nails, lymphedema and pleural effusion or pleuroparenchimal diseases such as bronchiectasis, recurrent lung infections or chronic cough. In most cases the prognosis is benign, however this syndrome was described in association with malignancy or is thought as an autosomal dominant inherited condition [3]. The diagnosis is underestimate: the triad is simultaneously present in only a quarter of patients with the syndrome [4] and often symptoms in the older population are referred to other diseases.

\section{CASE REPORT}

A 55-year-old man was admitted to our ward for short- ness of breath, productive cough and edema of the legs and feet. The patient was a smoker and had an history of recurrent pleurites with effusion, chronic venous insufficiency, psoriasis at palms of hands and at soles of feet. He noted the comparison, three years ago of marked thickening and yellow discoloration of the nails in hands and feet (Figures 1 and 2). During the last year he had a surgical treatment for nasal polyps. The electrocardiogram showed a left anterior hemiblock and an heart rate of 63 beats per minute (Figure 3), but the doppler echocardiographic study of ventricular function demonstrated normal size and motility of the left ventricle, a normal

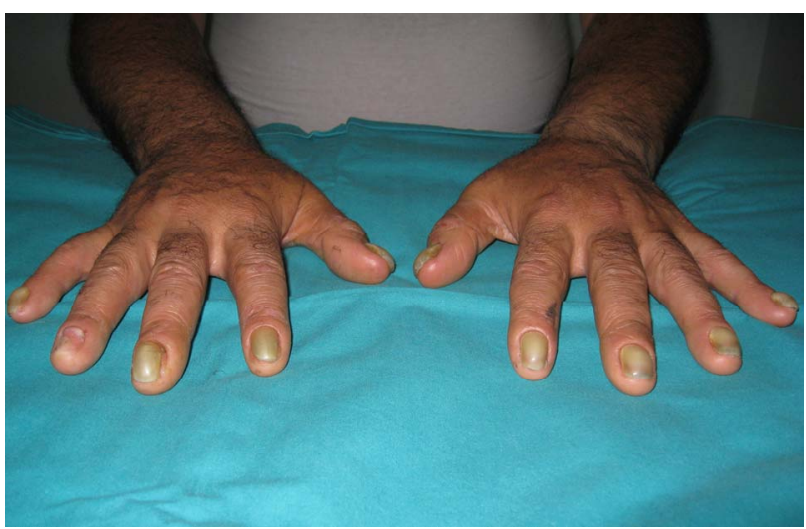

Figure 1. Image showing yellow nails and dystrophia.

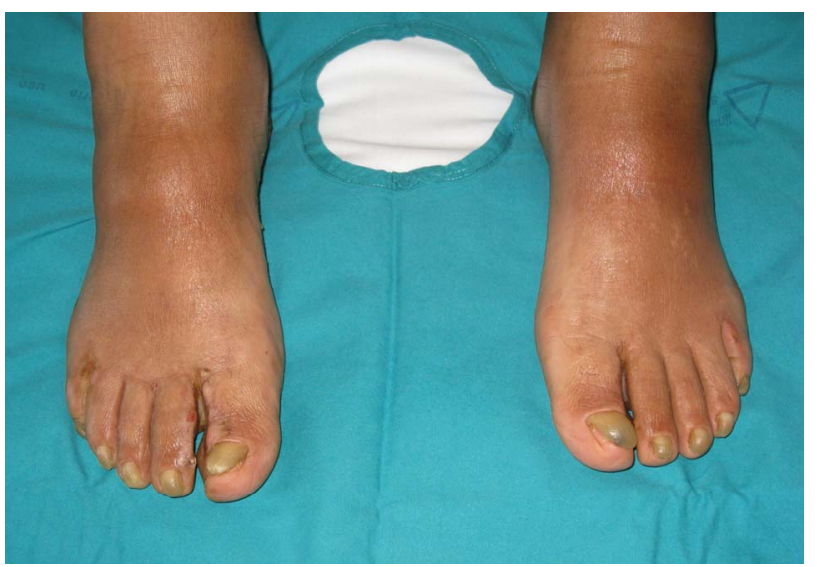

Figure 2. Bilateral yellow nails and parzially visible lymphedema of the feet. 
ejection fraction and no valvular steno-insufficiency. The chest X-ray showed a right-sided pleural effusion with parenchymal atelectasis. The second day a diagnostic thoracentesis was performed, removing $2.100 \mathrm{ml}$ of clear yellow fluid and a thoraco-abdominal computed tomography scan confirmed what X-ray showed, without further findings (Figure 4). Mantoux skin test was negative. Blood test screening upon admission was normal and the cytologic examination of pleural fluid revealed mesothelial cells with inflammatory changes. Pleurodesis was performed by thoracic surgeons on the right side: they removed $3.000 \mathrm{ml}$ of clear yellow fluid, withdrew multiple biopsies of parietal pleura and did a pleural talcage on the same side. The cytologic examination showed again mesothelial cells with inflammatory changes, but pleural biopsies revealed fibrosis, hyperplastic/reactive focal aspects of mesothelium with activated lymphoid follicles. The bacterial culture of the pleural fluid was negative for any kind of bacterium. The patient was discharged after 3 days with itraconazole (400 mg daily for 1 week per month, over a period of 9 month) [5], high dose vitamin E (1.200 IU daily for 2 weeks) and zinc supplements [6] as home therapy. The pleural drainage was removed at ambulatory monitoring 20 days later. The patient, at the moment, is at home in a fairly good condition, in follow-up by pneumologist.

\section{DISCUSSION}

\subsection{Diagnosis}

The diagnosis of Yellow Nail Syndrome is essentially clinical in nature and one of exclusion; is usually established by the classical triad: yellow discoloured nails, lymphedema and pleural effusion, but the presence of two of them has been judjed to be sufficient for diagnosis [7].

\subsection{Pathogenesis}

The pathogenic mechanism underlying this syndrome has not been defined, but hypoplasia of the lymphatic system as generalized phenomenon was suggested. A precipitating event is needed such as insect bites, erysipela or injury which increase the load on the already deficient lymphatics.

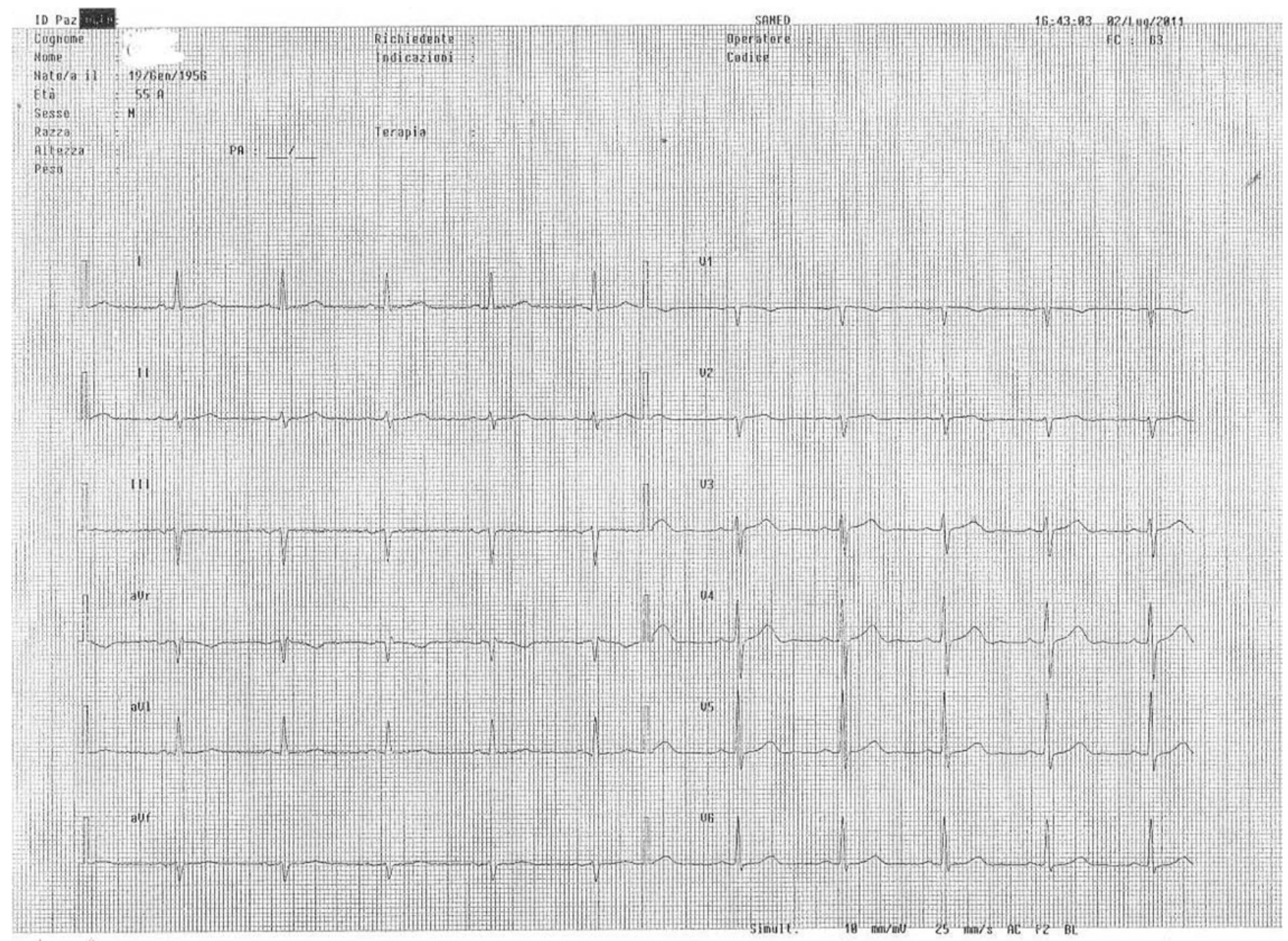

Figure 3. Electrocardiogram at the admission. 


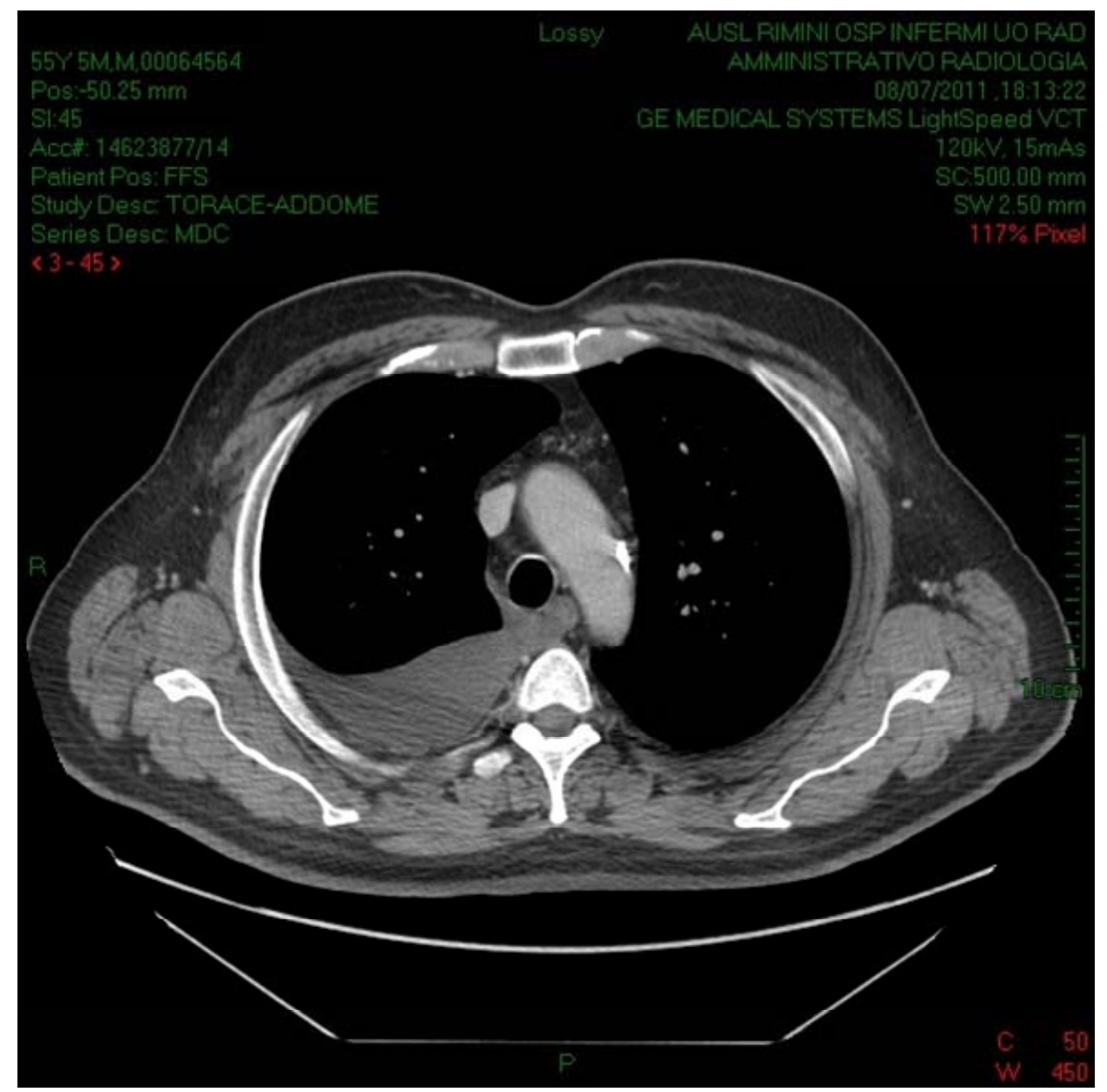

Figure 4. Computed tomography revealed pleural effusion.

\subsection{Symptoms and Signs}

Individual manifestations of the syndrome can appear from birth to late adult life and no gender predominance has been noted. This syndrome has been associated with autoimmune disorders, such as thyroiditis, systemic lupus erythematosus, and rheumatoid arthritis or tuberculosis, AIDS, diabetes mellitus, myocardial infarction and with the use of certain drugs such as gold, penicillamine, griseofulvin [8] or with malignancy such as cancer of the breast, larynx, lung [9]: yellow nails may be a paraneoplastic manifestation of cancer that may resolve with effective treatment. Yellow nails also are reported in several other conditions apart from yellow nail syndrome such as rheumatoid arthritis, non-Hodgkin's lymphoma, carcinoma gall bladder, melanoma, mycosis fungoides, diabetes mellitus or psoriasis. Our patient had an history of psoriasis: this disease produces a variety of changes in the appearance of finger and toe nails such as discolouring under the nail plate, pitting of the nails, lines going across the nails, thickening of the skin under the nail, and onycholysis.

\subsection{Therapy}

There's no well coded therapy for the yellow nail syndrome, but it improve in about one half of patients, often without therapy. Octreotide, a synthetic analogue of somatostatin, has an unclear mechanism of action; it acts by inhibiting lymph fluid excretion in the lymphatic vessels [10]. Patients need an optimal management of respiratory tract infections, but recurrent pleura effusions may require pleurodesis. Some experts propose vitamin $\mathrm{E}$ for the treatment of yellow nails, but the exact role of this vitamin remains unclear and the evidence is anedoctal [11]; itraconazole is expensive, difficult to tolerate, and is of questionable efficacy [5].

We think the diagnosis of Yellow Nail Syndrome is rarely achieved because symptoms are usually referred to other disease or syndromes.

\section{REFERENCES}

[1] Samman, P.D. and White, W.F. (1964) The "yellow nail” syndrome. British Journal of Dermatology, 76, 153-157. doi:10.1111/j.1365-2133.1964.tb14499.x 
[2] Emerson, P.A. (1966) Yellow nails, lymphedema and pleural effusion. Thorax, 21, 247-253. doi:10.1136/thx.21.3.247

[3] Razi, E. (2006) Familial yellow nail syndrome. Dermatology Online Journal, 12, 15.

[4] Nanda, S., et al. (2009) Yellow nail syndrome. Canadian Medical Association Journal, 181, 614. doi:10.1503/cmaj.080255

[5] Tosti, A., et al. (2002) Systemic itraconazole in the yellow nail syndrome. British Journal of Dermatology, 146, 1064-1067. doi:10.1046/j.1365-2133.2002.04671.x

[6] Maldonado, F., et al. (2009) Yellow nail syndrome. Current Opinion in Pulmonary Medicine, 15, 371-375. doi:10.1097/MCP.0b013e32832ad45a

[7] Hiller, E., et al. (1972) Pulmonary manifestations of the yellow nail syndrome. Chest, 61, 452-458.

\section{doi:10.1378/chest.61.5.452}

[8] Hershko, A., et al. (1997) Yellow nail syndrome. Postgraduate Medical Journal, 73, 466-468. doi:10.1136/pgmj.73.862.466

[9] Thomas, P.S., et al. (1987) Yellow nail syndrome and bronchial carcinoma [letter]. Chest, 92, 191. doi:10.1378/chest.92.1.191a

[10] Makrilakis, K., et al. (2004) Successful octreotide treatment of chylous pleural effusion and lymphedema in the yellow nail syndrome. Annals of Internal Medicine, 141, 246-247.

[11] Maldonado, F., et al. (2008) Yellow nail syndrome: Analysis of 41 consecutive patients. Chest, 134, 375-381. doi:10.1378/chest.08-0137 